\title{
Fuzzy Logic Based Fault Detection in Distributed Sensor Networks
}

\author{
L. B. Bhajantri
}

\author{
Department of Information Science and Engineering, Basaveshwar Engineering College, Bagalkot, Karnataka, India \\ *Corresponding Author: lokeshcse@yahoo.co.in, Tel.: +91-8904614106
}

Available online at: www.isroset.org

Received: 18/Mar/2018, Revised: 24/Mar/2018, Accepted: 02/Apr/2018, Online: 30/Apr/ 2018

\begin{abstract}
Distributed Sensor Networks (DSNs) are emerging as a promising area in the real world environment. The nodes are deployed randomly in the DSN environment. The deployed nodes are leads to failure of nodes due to certain environmental conditions. The failure nodes are causes the packet dropping from source node to sink node or disconnection of network. The faulty nodes are also causes the degradation of Quality of Service (QOS) of the network. This paper proposes a fuzzy logic based efficient fault detection for routing in DSNs. This approach incorporates the performance parameters of the network in terms of energy consumption, throughput, and latency.
\end{abstract}

Keywords—Distributed Sensor Network, Fuzzy Logic, Fuzzy Inferences, and Fuzzy Membership.

\section{INTRODUCTION}

Distributed Sensor Network (DSN) has set of intelligent distributed sensor nodes with high speed. The nodes in the DSN is assumed to be large number of homogeneous or heterogeneous nodes, those nodes are scattered logically, spatially or geographical over an environment. The distributed sensor nodes are used to collect the data from the respective environments logically, such data is processed to sink node through its intermediate node in the network periodically. The sensor nodes are used in different application for collecting and processing of data over the network. The exploitation of nodes are varies with the applications [1] [2]. The use of nodes are able to acquire and processing of data and performing the computational tasks in DSNs. Such network has the prospective to allow wide range of applications and faces major challenges like frequent network disconnection, limited resources, limited computational, and memory [3]. Because of these conditions an efficient and effective procedure for detecting faulty nodes from the DSN is challenging task. The DSN also faces the following changes as compared to traditional network are: energy aware and efficient, exception free and self configuring, and must respond to dynamic environment, data-centric and data concentrated and application specific, and fault tolerance [4].

Sensor node are scattered in hostile and unattended environments. Such deployed sensor nodes are failure due to certain factors in DSN like fire or extreme heat, animal or vehicular accidents, malicious activity, or by extended use, devices positions over time, possibly disconnecting the network. There are various levels of faults in DSN environment such as: at node level or sink node level and network level faults (link failure and packet error). In this work, it has been considered that a node level fault. The sensor nodes are works on the basis of battery power i.e., energy. So that energy is one of the major challenging resources for sensing and processing of data in DSN [5]. Fuzzy logic control is capable of making real time decisions, even with incomplete information. The proposed scheme is simulated using fuzzy logic system for detecting efficient failure of nodes to avoid the degradation of network performance in advance for transmission of data.

The rest of the paper is organized as follows. The related work towards the proposed work is presented in section II. The put forward work for detection of failure nodes and data processing using fuzzy logic is depicted in Section III. The section IV describes the simulation results and discussions and finally concludes the work in section V.

\section{RELATED WORK}

The following related works are discussed towards the proposed scheme: the work given in [6] describes the fuzzy logic based management of faults in wireless sensor networks. This scheme incorporates the fault detection, isolation, and recovery algorithms based cluster based framework. The work given in [7] depicts the faulty reading detection in wireless sensor networks using fuzzy logic. Fault detection and routing in wireless sensor network is discussed in [8]. The fault detection method in wireless sensor 
networks is described in [9]. This scheme proposes the distributed fault detection method for failure nodes in wireless sensor networks. The simulation method describes the performance of the distributed fault detection method for fault detection accuracy greatly. The work given in [10] presents the energy aware fault tolerance for routing in wireless sensor networks. The $k$-Vertex Disjoint Path Routing (kVDPR) algorithm is resented for routing. A fuzzy rule based faulty node classification and management scheme is presented in [11]. Also it has presented the efficient data routing algorithm for transmission of data over the faulty nodes in the network.

The work given in [12] describes the different fault detection techniques and provides a new taxonomy to integrate new fault detection techniques. Also discusses the shortcomings, advantages and future research directions or fault detection in wireless sensor networks. Fault tolerance in DSNs using Bayesian networks is depicted in [13]. This work identifies the faults like node, link, and sink node. This scheme uses the Bayesian model to achieve the fault tolerance of the network for providing the services to the users. Some of the related works are given in [14] [15] [16].

In generally, most of the works are considered in the form centralized approach for fault detection in the network. The centralized approach causes the massive volume message traffic and past energy depletion in certain regions of the network. In this work, consider the distributed approach to detection of faults efficiently. In this scheme, each sensor node takes the decisions at certain levels before communicating with the sink node of the DSN. The node can itself find the faulty information and coordinate with their neighbors to detect and identify the network faults before communicating with the central node or sink node.

\section{PROPOSED WORK}

The objective of fault detection is to verify that the services being provided for functioning continuously to the users. The proposed system consists of set of distributed nodes are as shown in figure1. The numbers of sensor nodes are deployed randomly in the real time environment. The deployed sensor nodes are assumed to be stationary with initial amount of energy. The failures of sensor node are common in the network, but handling of failure node is a biggest challenging task. The failure could be node failure or link failure. The failure nodes are causes the degradation of network performance. Hence, it is required that network failures are detected in advance and appropriate measures are taken to sustain network operation.

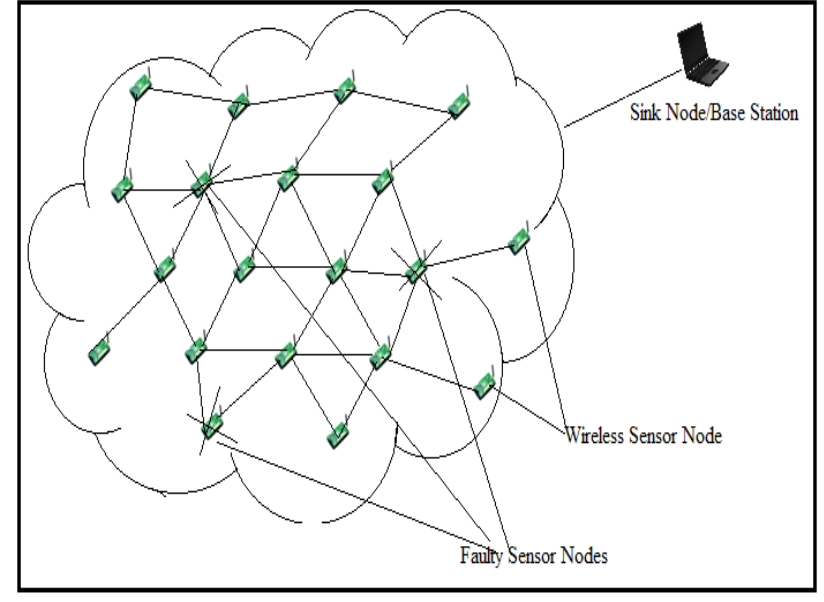

Figure1. System Architecture.

The proposed fuzzy logic algorithm is used to efficiently detect the faults in an advance before transmitting the data from source node to sink node. The proposed work has two stages of fault tolerance in the DSN. The first stage is the fault detection stage, which is used to identify the failure nodes and link failure between the nodes in the DSN. Another stage is the fault recovery, which is re-initializes the network with active nodes to establish path between sources and sink node. Finally, sink node performs the action upon receiving the information form sensor nodes.

The figure2 depicts the communication model in DSNs. The communication model provides the continuous data transmission from source to sink node in presence of faulty nodes in the DSNs. In this model, each sensor node sends the heartbeat message to its neighbor nodes periodically or regular intervals to indicate its active nodes. Each sensor node doesn't receive the heartbeat message from its neighbor nodes, so that sensor node assumes any of the neighbor nodes is failure. Each sensor node maintains the neighbor node informations like energy level, bandwidth, and link efficiency information between the nodes; based on these information each node detects the faulty nodes within its neighbors by using fuzzy logic in the network. In this section, fuzzy logic and inferences, functioning scheme, and fuzzy membership functions are described. 


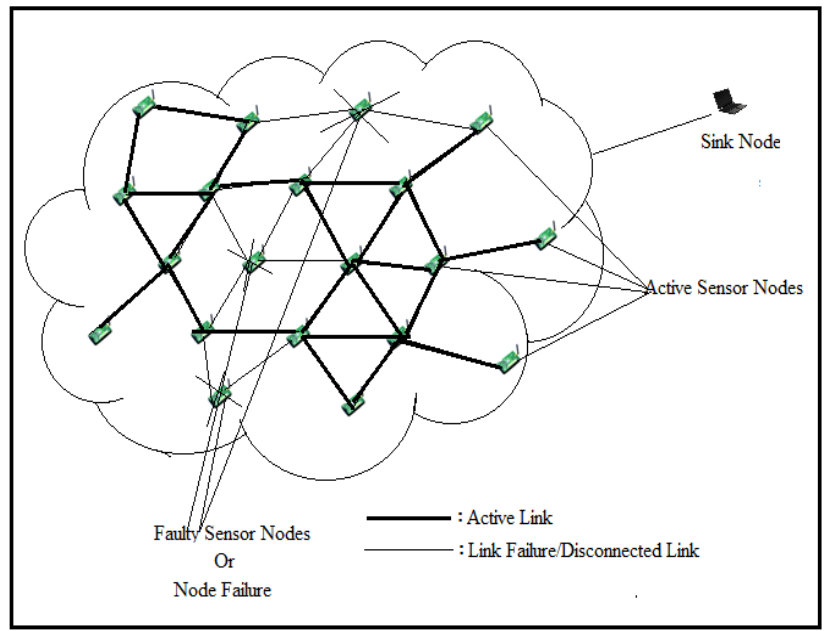

Figure2. Communication Model in DSNs.

\section{A. Proposed Fuzzy Logic}

Fuzzy Logic System (FLS) is a general non-linear inputoutput mapping [17] method. FLS operates with fuzzy a set that extends the notion of crisp sets. A fuzzy set is characterized by a membership function. The fuzzy systems are associated with the following four core components: (1) the fuzzifier - it takes crisp inputs into fuzzy sets by using the membership functions, (2) the fuzzy rules - which are expressed in the collection of IF-THEN statements, having fuzzy propositions as antecedents and consequences, (3) the fuzzy inferences - the fuzzy inference engine combines the fuzzy rules to obtain an aggregated fuzzy output, and (4) finally, the de-fuzzifier maps the fuzzy output back to a crisp number that can be used for making decisions [18].

\section{B. Proposed Fuzzy Inferences}

The given fuzzy inferences are used in the process of deriving logical conclusion from premises known or assumed to be true. Let di be the distance between nodes in the network. It can be computed by using Euclidian Distance Formula (EDF) given by Eq. (1) [19].

$$
\mathrm{d}_{\mathrm{i}}=\sqrt{\left(x_{1}-x_{2}\right)^{2}+\left(y_{1}-y_{2}\right)^{2}}
$$

Link efficiency can be computed as follows. Let $C_{i}$ be capacity of a discrete-time discrete-valued channel, ' $\mathrm{B}$ ' be the bit rate $(\mathrm{Hz})$ of a channel, ' $\mathrm{E}_{\mathrm{T}}$ ' be the total energy consumed for transmission of a bit in link 'i', SNR be the signal-to-noise ratio [20]. Capacity of channel ' $i$ ' is:

$$
\mathrm{C}_{\mathrm{i}}=\mathrm{B} \log _{2}(1+\mathrm{SNR})
$$

Assume ' $\mathrm{E}_{\mathrm{N}}$ ' stands for energy consumption for the transmission of the packets. ' $\mathrm{E}_{\mathrm{N}}$ ' can be computed by using Eq. (3):

$$
\mathrm{E}_{\mathrm{N}}=\mathrm{S}_{\mathrm{E}} \times \mathrm{P}_{\mathrm{i}} / \text { bits }+\mathrm{T}_{\mathrm{E}} \times \mathrm{P}_{\mathrm{i}} / \text { bits }
$$

Where,

$\mathrm{S}_{\mathrm{E}}$ - energy required for sensing data or packets,

$\mathrm{T}_{\mathrm{E}}-$ energy required for transmission of data or packets,

$\mathrm{P}_{\mathrm{i}}-$ size of packets in terms of bits.

Let ' $E$ ' would be the energy consumed for the transmission of a bit per distance $d_{i}$. The energy of node ' $E$ ' is [21]:

$$
\mathrm{E}=\mathrm{E}_{\mathrm{N}} \times \mathrm{d}_{\mathrm{i}}
$$

Assume ' $\mathrm{L}_{\mathrm{eff}}$ ' is the link efficiency for the nodes in the network:

$$
\mathrm{L}_{\mathrm{eff}}=\mathrm{C}_{\mathrm{i}} / \mathrm{E}
$$

Let ' $R_{E}$ ' be the residual energy of each node in the DSNs as follows:

Where,

$$
\mathrm{R}_{\mathrm{E}}=\mathrm{I}_{\mathrm{E}}-\mathrm{E}_{\mathrm{i}}
$$

$\mathrm{I}_{\mathrm{E}}=$ initial energy of node, $\mathrm{E}_{\mathrm{N}}=$ energy consumption.

Based on above equations the inferences were derived for the proposed work is given in Table I and Table II.

\section{Functioning Scheme}

Algorithm for Cluster Head selection and Data Processing Input:
$D_{N}:$ A Distributed Senor Network
$S_{i}:$ A sensor node of DSNs
$E_{N}$ : Energy of sensor node
$L_{\text {eff }}$ : A link efficiency of sensor nodes
$S_{N}:$ Number of Sink node
ThE: Threshold Level Energy
ThLeff: Threshold Level Link Efficiency

Output:

Selection of efficient fault detection in the DSNs Number $\left(F\left(S_{i}\right)\right)==$ True)

Functions:

fuzzylogic $\left(\mathrm{L}_{\text {eff, }}, \mathrm{S}_{\mathrm{i}}\right)$;

Send (data, $S_{i}$ );

Receive $\left(S_{i}, S_{N}\right)$;

Procedure:

1. Deploy the number of sensor nodes randomly;

2. for $(\mathrm{i}=0 ; \mathrm{i}<\mathrm{N} ; \mathrm{i}++)$

if $\left(E_{N} \geq T h E\right.$ and Leff $\geq$ ThLeff $)$$$
\text { Else }
$$

Node is Active Node;

Node is Dead Node; endif

endfor

3. Data Transmission or processing over the nodes in the DSN is as follows:

if $\left(\left(\mathrm{S}_{\mathrm{i}}\right)=\right.$ TRUE $)$

$$
\text { Data }=\text { fuzzylogic }\left(\mathrm{L}_{\text {eff, }}, \mathrm{S}_{\mathrm{i}}\right)
$$

else

Select alternate path with better link efficiency; endif

Table I. Fuzzy If- Then Rules for Faulty Node Detection 


\begin{tabular}{|c|c|c|}
\hline $\begin{array}{c}\text { Residual Energy of } \\
\text { Sensor Node }\end{array}$ & Link Efficiency & Result of Node Failure \\
\hline High & High & Very Low \\
\hline High & Medium & Average \\
\hline High & Low & Average \\
\hline Medium & High & Average \\
\hline Medium & Medium & Low \\
\hline Medium & Low & High \\
\hline Low & High & Average \\
\hline Low & Medium & High \\
\hline Low & Low & Very High \\
\hline
\end{tabular}

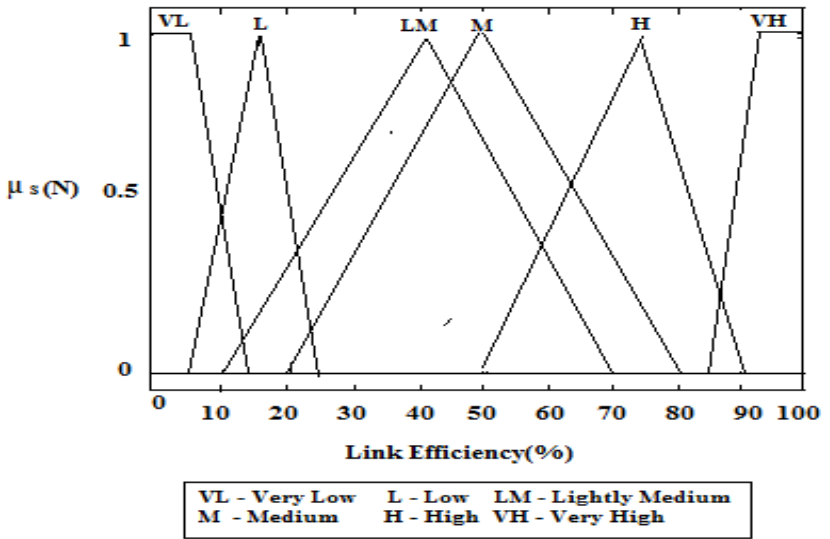

\begin{tabular}{|c|c|c|}
\hline $\begin{array}{c}\text { Energy } \\
\text { Level of Nodes }\end{array}$ & $\begin{array}{c}\text { Link efficiency } \\
\text { between sensor } \\
\text { nodes }\end{array}$ & $\begin{array}{c}\text { Results for data } \\
\text { processing }\end{array}$ \\
\hline High & High & Very High \\
\hline High & Medium & High \\
\hline High & Low & Medium \\
\hline Medium & High & High \\
\hline Medium & Medium & Medium \\
\hline Medium & Low & Medium \\
\hline Low & High & Medium \\
\hline Low & Medium & Medium \\
\hline Low & Low & Low \\
\hline
\end{tabular}

\section{Fuzzy Membership Functions}

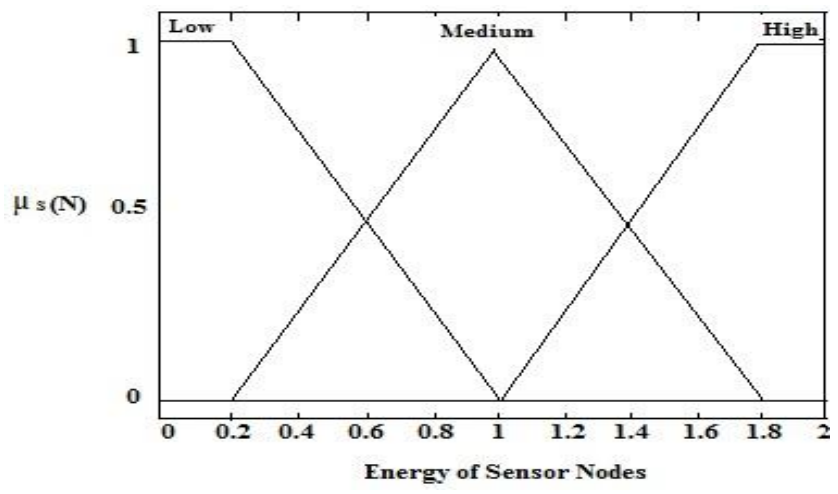

Figure 3. Fuzzy Membership Function of Energy.

The membership function for the proposed work is given below:

$$
\mu_{S}(N)=\left\{\begin{array}{lll}
1 & \text { if } N=s_{;} & \text {Active Sensor node } \\
0 & \text { if } N \neq s_{3} & \text { Dead node }
\end{array}\right.
$$

$\mu_{\mathrm{S}}(\mathrm{N})$ is a mapping of $\mathrm{N}$ on $[0,1]$ i.e., degree of belonging of some node $S_{i}$ to the universe $S_{i}$ can be member $0 \leq \mu_{S}(\mathrm{~N}) \leq 1$. The fuzzy membership functions and some of the existing rules, which are used in the fuzzy inference system are shown in figure 3 and figure4 and table I and table II respectively. 
Size of packets $(\mathrm{P})=$ 64Bits, 128Bits, 512Bits, 1024Bits and so on, and Threshold Level Energy (THE) $=0.05$ Joules, Transmission of data $=$ bits $/ \mathrm{sec}$ and bandwidth of the network $(\mathrm{BN})=4 \mathrm{mbps}$.

\section{Performance Parameters}

The following performance parameters were used in proposed scheme:

1) Throughput: It is the ratio of number of packets sent to the number of packets received successfully.

2) Energy consumption: As the number of nodes increases, the energy of each node increases with respect to fault detection ratio (FDR) for routing of data in the DSN.

3) Latency: As the number of nodes increases, the increases the delay of network for transmission of data with respect to FDR.

\section{Results and Discussions}

The figure5 depicts the throughput of the network with the number of nodes. As the number of nodes increase, the gradully decrease in the throughput of the network with respect to fault detection ratio (FDR) because of more communication overhead or congestion between the nodes. In this scheme, we considered the FDR ratio of the network is as follows: $25 \%, 50 \%$, and $75 \%$. If FDR is more in the network, to achieve better throughput because the proposed scheme is simulted using distributed approach for fault detection in advance for transmission of data over the network.

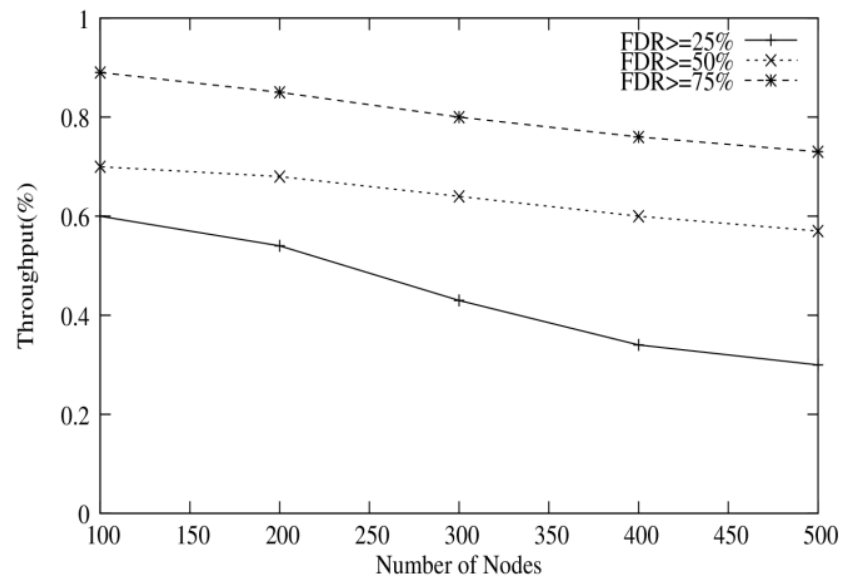

Figure 5. Number of Nodes vs. Throughput.

The latency of the network with respect to number of nodes is as shown in figure6. The increase the number of nodes in the network that leads to increases the delay for transmission of data. Because of increase the number of nodes causes the communication overhead. If the FDR is efficient in the network, the delay is less between the sensor nodes for transmission of data from source node to sink node in the DSNs. The proposed fuzzy based fault detection algorithm detects the faults efficiently to avoid the delay or latency for communication overhead.

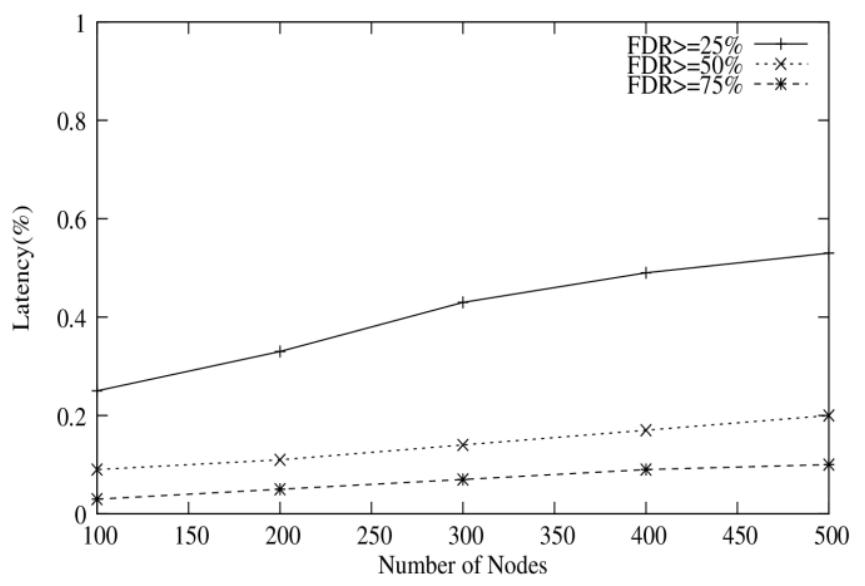

Figure6. Number of Nodes vs. Latency.

The figure7 describes the number of nodes increases in the DSNs, there is a increase in an energy consumption in the sensor nodes. Initial deployment of sensor nodes are assumed to be good number of active nodes in the network, as the percentage of FDR increases, the proposed algorithm utilizes less energy consumption for data transmission in the DSNs.

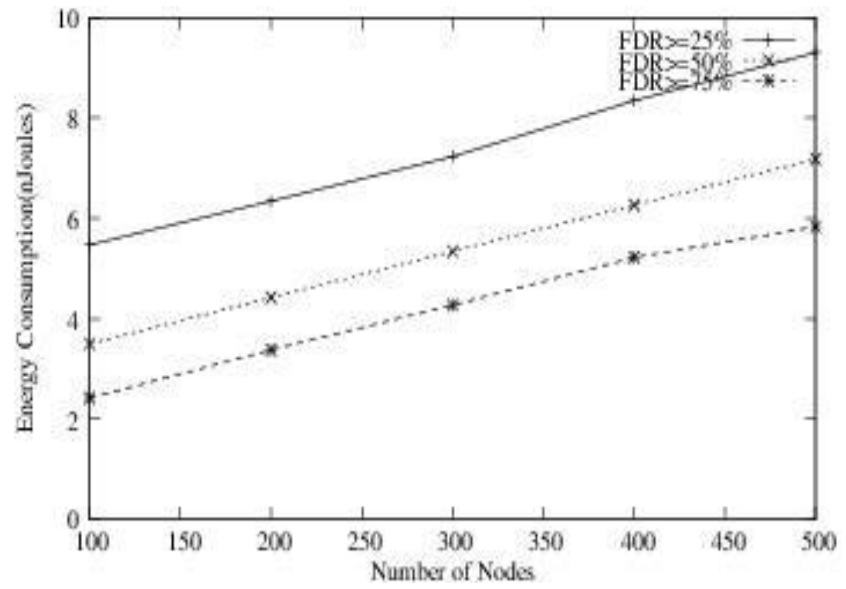

Figure 7. Number of Nodes vs. Energy Consumption.

\section{CONCLUSION}

The proposed method is energy efficient and responsive to network. It includes efficient detection of faulty nodes and data processing using fuzzy logic for routing. It has been observed that fuzzy logic is used to probabilistically select an efficient detection of failure nodes to achieve the better results. It has been observed that the performance of proposed algorithm robust and fast. The proposed scheme is simulated in terms of performance parameters such as throughput, latency, and energy consumption.

\section{REFERENCES}


[1] S., S, Ankit. T, Brooks. R., R, "An Overview of Distributed Sensors Network".

Available:

http://books.google.com/books/about/Distributed-sensor-networks.ht ml?id=Nff5

[2] S. S, Iyengar. S., S, "Taxonomy of Distributed Sensors Networks". Available:

http://systems.ihpmicroelectronics.com/uploads/downloads/DS1_2007 _ 06_Taxonomy.pdf.

[3] Q. Hairong, S. S., Iyengar,I, C. Krishnendu, "Distributed Sensor Networks- A Review of Recent Research", Journal of Distributed Sensor Networks for Real Time System with Adaptive Configuration, Vol. 338, No, 6, pp. 655 - 668, 2007.

[4] Y. C., Chong, P. S., Kumar, "Sensor Networks: Evolution, Opportunities, and Challenges", In Proceedings of the IEEE, Vol. 91, No. 8, pp. 1247 - 1256, 2003.

[5] L. Paradis, Q. Han, "A Survey of Fault Management in Wireless Sensor Networks", Journal of Network and Systems Management, Vol. 15, No. 2, pp. 171 - 190, 2007.

[6] S. Chang, T. Huang, "A Fuzzy Knowledge Based Fault Tolerance Algorithm in Wireless Sensor Networks", In proceedings of International Conference on Advanced Information Networking and Applications, pp.891-896, 2012.

[7] S. J., Datgheib, “An Efficient Approach to Detect Faulty Readings in Long-thin Wireless Sensor Network using Fuzzy Logic", In proceedings of Future Communication Networks, pp. 88-92, 2012.

[8] I. Banerjee, P. Chanak, H. Rahaman, T. Samanta, "Effective Fault Detection and Routing Scheme for Wireless Sensor Networks", Journal of Computers and Electrical Engineering, vol.40, pp.291-306, 2014.

[9] P.Jiang, "A new Method for Node Fault Detectionin Wireless Sensor Networks", Journal on Sensors, vol.9, pp.1282-1294, 2009.

[10] P. Chanak, I. Banerjee, R. S., Sherratt, "Energy-Aware Distributed Routing Algorithm to Tolerate Network Failure in Wireless Sensor Networks", Journal of Adhoc Networks, vol.56, pp. 158-172, 2017.

[11] P. Chanak, I. Banerjee, "Fuzzy Rule Based Faulty Node Classification and Management Scheme for Large Scale Wirless Sensor Networks", Journal of Expert Systems with Applications, vol.45, pp. 307-321, 2017.

[12] T. Muhammed, R. A., Shaikh, "An Analysis of Fault Detection Strategies in Wireless Sensor Networks", Journal of Network and Computer Applications, vol.78, pp. 267-287, 2017.

[13] L.B., Bhajantri, N.Nalini, "Bayesian Network Based Fault Tolerance in Distributed Sensor Networks", Journal of Telecommunications and Information Technology, vol.3, pp.1-9, 2014

[14] Jin-Shyan. L, Wei-Liang. C, "Fuzzy-Logic-Based Clustering Approach for Wireless Sensor Networks Using Energy Predication, IEEE Journal of Sensors, vol.12, no.9, pp. 281-2897, 2012.

[15] Mourad. H, Hervé. G, "A Routing Algorithm based on Fuzzy Logic Approach to Prolong the Lifetime of Wireless Sensor Networks", International Journal of Open Scientific Research, vol.1, no.5, pp. 2435, 2013.

[16] J. Shell, S. Coupland, E. Goodyer, "Fuzzy Data Fusion for Fault Detection in Wireless Sensor Networks", In proceedings of workshop on Computational Intelligence, pp. 1-6, 2010.

[17] M. Rogaia, A. Nauman, P. William, C. Frank, "An Energy Efficient Fuzzy Logic Cluster Formation Protocol in Wireless Sensor Networks", In proceedings of 3rd International Conference on Ambient Systems, Networks and Technologies, vol.10, pp. 255 - 262, 2012.

[18] J. Maryam, H. T., Abolfazl, “A Routing Algorithm based on Fuzzy Clustering and Minimum Cost Tree (FCMCT) in Wireless Sensor
Network" Journal of Advances in Computer Research, vol.5, no.3, pp. 113-123, 2014.

[19] S. Nishant, S. Upinderpal, "A Location Based Approach to Prevent Wormhole Attack in Wireless Sensor Networks", International Journal of Advanced Research in Computer Science and Software Engineering, vol.4, no.1, pp. 840-845, 2014.

[20] T. Herbert, L. Donald, "Schilling Principles of Communication, Systems", McGraw-Hill 1986.

[21] G. Miao, N. Himayat, Y. G., Li, "Energy Efficient Link Adaptation in Frequency Selective Channels", IEEE Transaction on Communication, vol.58, no.2, pp. 2010.

[22] W. Heinzelman, A. Chandrakasan, H. Balakrishnan, "Energy Efficient Communication Protocol for Wireless Micro Sensor Networks", In the proceedings of the IEEE Hawai International conference on system sciences, vol.8, pp.8020-8030,2000.

Authors Profile
Lokesh B. Bhajantri received
P.h.D (Computer Science and
Engineering) degree from
Visveswaraiah Technological
University (VTU), Belgaum,
Karnataka, 2015. He is presently
working as a Associate Professor
in the Department of Information

Basaveshwar Engineering college, Bagalkot, India, He has experience of around 13 years in teaching and research. His areas of interest include Vehicular Adhoc Networks (VANETs), Vehicular Cloud Computing (VCC), Wireless or Distributed Sensor Networks (DSNs), Semantic Sensor Web (SSW), Cloud Computing, Ubiquitous Networks, Cognitive Internet of Things (CIoT), e-commerce, u-commerce, mobile computing and communications, networking protocols, genetic algorithms, applications of agents and real time systems. He has given invited lectures in AICTE sponsored workshops and seminars. He has published chapter in Handbook of Research on Telecommunications Planning and Management for Business IGI Global, 12 referred international conferences papers and 10 referred international Journals. He is a reviewer of some journals and conferences. $\mathrm{He}$ is a member of Board of Studies (BOS) of Department of Information Science and Engineering, Basaveshwar Engineering College, Bagalkot, Karnataka, India. He is a member of International Association of Computer Science and Information Technology (IACSIT). He is guest editor of special issue on Advances in Cloud and Internet of Things [ISSN: 2376-7715 (Print)], Science PG Publishers. 